sciendo

\section{TATRA \\ MOUNTaiNS \\ Mathematical Publications}

DOI: $10.2478 / \mathrm{tmmp}-2021-0024$

Tatra Mt. Math. Publ. 79 (2021), 135-148

\title{
PROPERTIES OF THE KATUGAMPOLA FRACTIONAL OPERATORS
}

\author{
BARBARA ŁUPIŃSKA
}

Insitute of Computer Science of University of Białystok, POLAND

\begin{abstract}
In this work, there are considered higher order fractional operators defined in the sense of Katugampola. There are proved some fundamental properties of the Katugampola fractional operators of any arbitrary real order. Moreover, there are given conditions ensuring existence of the higher order Katugampola fractional derivative in space of the absolutely continuous functions.
\end{abstract}

\section{Introduction}

In 2011 Udita N. Katugampola introduced (see [1, [2]) new fractional operators, which were named after his surname, i.e., the Katugampola fractional integral and the Katugampola fractional derivative. Such operators depend on some extra parameter $\rho>0$, which by taking $\rho \rightarrow 0^{+}$reduce to the Hadamard fractional operators, and for parameter $\rho=1$ become the Riemann-Liouville fractional operators. Thanks to this, the use of the Katugampola fractional operators simplifies the theory. If we prove something for the Katugampola derivative, we get this fact for both the Riemann-Liouville derivative and the Hadamard derivative.

Nowadays, these operators are gaining more and more popularity. The Katugampola fractional derivative is widely discussed in the literature. They have applications in fields such as probability theory [3], theory of inequalities [4,5], differential equations [6, 7], Mellin transforms [8], maximum principle [9] and chaos and stability [10]. So far, in the literature, the Katugampola fractional operators occur only up to the order 2 . In this work, the author is interested in the Katugampola derivative of the higher order. There is considered the existence of the Katugampola fractional derivative of an arbitrary real order $\alpha>0$.

(C) 2021 Mathematical Institute, Slovak Academy of Sciences.

2010 Mathematics Subject Classification: 26A33, 34A08.

Keywords: fractional calculus, Katugampola fractional operators, higher order, existence.

(C) (1) $\Theta$ Licensed under the Creative Commons BY-NC-ND 4.0 International Public License. 


\section{BARBARA ŁUPIŃSKA}

Moreover, in the last section, there are described some useful properties of these operators, which can be used to further research on the subject, where is still much to be done.

\section{Preliminaries}

In this paper, we denote by $\mathbb{N}$ a set of natural numbers $\mathbb{N}=\{1,2, \ldots\}$ and $\mathbb{N}_{0}=\{0,1,2, \ldots\}$.

In this section, we give the definitions of the Katugampola fractional integrals and fractional derivatives on a finite positive interval of the real line $[a, b](0<$ $a<b<\infty)$ with examples.

To formulate it, we need the following special functions:

- Gamma function defined for $x>0$

$$
\Gamma(x)=\int_{0}^{\infty} t^{x-1} e^{-t} d t,
$$

which, for $n \in \mathbb{N}$, has the following properties

$$
\begin{aligned}
& \Gamma(n)=(n-1) !, \\
& \Gamma(n)=n \Gamma(n-1) .
\end{aligned}
$$

- Beta function defined for $x, y>0$

$$
B(x, y)=\int_{0}^{1} t^{x-1}(1-t)^{y-1} d t
$$

which can be written as

$$
B(x, y)=\frac{\Gamma(x) \Gamma(y)}{\Gamma(x+y)} .
$$

Moreover, let $L[a, b]$ be space of integrable functions $f:(a, b) \rightarrow \mathbb{R}$ such that

$$
\int_{a}^{b}|f(t)| d t<\infty .
$$

Definition 2.1. Let $n \in \mathbb{N}, n-1<\alpha<n, \rho>0,0<a<b<\infty$. The operators

$$
\begin{aligned}
& I_{a+\rho}^{\alpha, \rho} f(t)=\frac{\rho^{1-\alpha}}{\Gamma(\alpha)} \int_{a}^{t} \frac{\tau^{\rho-1}}{\left(t^{\rho}-\tau^{\rho}\right)^{1-\alpha}} f(\tau) d \tau, \\
& I_{b-\rho}^{\alpha, \rho} f(t)=\frac{\rho^{1-\alpha}}{\Gamma(\alpha)} \int_{t}^{b} \frac{\tau^{\rho-1}}{\left(\tau^{\rho}-t^{\rho}\right)^{1-\alpha}} f(\tau) d \tau,
\end{aligned}
$$




\section{PROPERTIES OF THE KATUGAMPOLA FRACTIONAL OPERATORS}

for $t \in(a, b)$ are called the left-sided and right-sided Katugampola integrals of fractional order $\alpha$, provided they exist.

In particular,

$$
I_{a+}^{\alpha, 1} f(t)=\frac{1}{\Gamma(\alpha)} \int_{a}^{t} \frac{f(\tau)}{(t-\tau)^{1-\alpha}} d \tau
$$

(Riemman-Lioville fractional integral)

$\lim _{\rho \rightarrow 0^{+}} I_{a+}^{\alpha, \rho} f(t)=\frac{1}{\Gamma(\alpha)} \int_{a}^{t}\left(\log \frac{t}{\tau}\right)^{\alpha-1} f(\tau) \frac{d \tau}{\tau} \quad$ (Hadamard fractional integral), and

$$
I_{a+}^{n, 1} f(t)=\int_{a}^{t} \int_{a}^{t_{1}} \int_{a}^{t_{2}} \ldots \int_{a}^{t_{n-1}} f(\tau) d \tau d t_{n-1} \ldots d t_{1}, \quad n \in \mathbb{N} .
$$

Let us introduce the notation $\delta_{\rho}$ for $\delta_{\rho}$-derivative defined by $\delta_{\rho}:=t^{1-\rho} \frac{d}{d t}$. We can easily prove that for $n \in \mathbb{N}$

provided $I_{a+}^{n, \rho} f$ exists.

$$
\delta_{\rho}^{n} I_{a+\rho}^{n, \rho} f(t)=f(t)
$$

Definition 2.2. Let $n \in \mathbb{N}, \rho>0,0<a<b<\infty, n-1<\alpha<n$. The operators

$$
\begin{gathered}
D_{a+}^{\alpha, \rho} f(t)=\delta_{\rho}^{n} I_{a+}^{n-\alpha, \rho} f(t) \\
D_{b-}^{\alpha, \rho} f(t)=(-1)^{n} \delta_{\rho}^{n} I_{b-}^{n-\alpha, \rho} f(t)
\end{gathered}
$$

for $t \in(a, b)$ are called the left-sided and right-sided Katugampola derivatives of fractional order $\alpha$, provided they exist.

In particular, for $\rho=1$ and for $\rho \rightarrow 0^{+}$

$$
\begin{aligned}
D_{a+}^{\alpha, 1} f(t) & =\left(\frac{d}{d t}\right)^{n} \frac{1}{\Gamma(n-\alpha)} \int_{a}^{t} \frac{f(\tau)}{(t-\tau)^{\alpha-n+1}} d \tau, \\
\lim _{\rho \rightarrow 0^{+}} D_{a+}^{\alpha, \rho} f(t) & =\frac{1}{\Gamma(n-\alpha)}\left(t \frac{d}{d t}\right)^{n} \int_{a}^{t}\left(\log \frac{t}{\tau}\right)^{n-\alpha-1} f(\tau) \frac{d \tau}{\tau},
\end{aligned}
$$

we obtain the Riemman-Liouville fractional derivative and the Hadamard fractional derivative, respectively. Moreover,

$$
D_{a+}^{0, \rho} f(t)=D_{b-}^{0, \rho} f(t)=f(t),
$$


and when $\alpha=n \in \mathbb{N}$, then

$$
D_{a+}^{n, \rho} f(t)=\delta_{\rho}^{n+1} I_{a+}^{1, \rho} f(t)=\left(t^{1-\rho} \frac{d}{d t}\right)^{n+1} \int_{a}^{t} \tau^{\rho-1} f(\tau) d \tau=\delta_{\rho}^{n} f(t)
$$

and, analogously,

$$
D_{b-}^{n, \rho} f(t)=(-1)^{n} \delta_{\rho}^{n} f(t)
$$

Now, we present the examples which show that Katugampola fractional integral and differential operators of power functions return power functions.

EXAMPLE. If $\alpha>0, \rho>0$ and $\lambda>-1$ then

$$
\begin{aligned}
& I_{a+}^{\alpha, \rho}\left(\frac{t^{\rho}-a^{\rho}}{\rho}\right)^{\lambda}=\frac{\Gamma(\lambda+1)}{\Gamma(\lambda+\alpha+1)}\left(\frac{t^{\rho}-a^{\rho}}{\rho}\right)^{\alpha+\lambda}, \\
& I_{b-}^{\alpha, \rho}\left(\frac{b^{\rho}-t^{\rho}}{\rho}\right)^{\lambda}=\frac{\Gamma(\lambda+1)}{\Gamma(\lambda+\alpha+1)}\left(\frac{b^{\rho}-t^{\rho}}{\rho}\right)^{\alpha+\lambda} .
\end{aligned}
$$

ExAmple. For $\rho>0, \alpha>0, \lambda>\alpha-1$, we have

$$
D_{a+}^{\alpha, \rho}\left(\frac{t^{\rho}-a^{\rho}}{\rho}\right)^{\lambda}=\frac{\Gamma(\lambda+1)}{\Gamma(\lambda+1-\alpha)}\left(\frac{t^{\rho}-a^{\rho}}{\rho}\right)^{\lambda-\alpha}
$$

and

$$
D_{b-}^{\alpha, \rho}\left(\frac{b^{\rho}-t^{\rho}}{\rho}\right)^{\lambda}=\frac{\Gamma(\lambda+1)}{\Gamma(\lambda+1-\alpha)}\left(\frac{b^{\rho}-t^{\rho}}{\rho}\right)^{\lambda-\alpha} .
$$

The equality (5) was proved in [14]. Formula (6) can be proved similarly by using substitution of the form $u=\frac{b^{\rho}-\tau^{\rho}}{b^{\rho}-t^{\rho}}$.

From the above, we have the following remark.

Remark 1. If $\alpha>0, n=[\alpha]+1$ and $\rho>0$, then

$$
D_{a+}^{\alpha, \rho}\left(\frac{t^{\rho}-a^{\rho}}{\rho}\right)^{\alpha-j}=0, \quad \text { for } j=1, \ldots, n .
$$

In this work, we are interested in the Katugampola fractional derivatives of order $\alpha \in(n-1, n], n \in \mathbb{N}$. Therefore, in the following, we give conditions ensuring existence of the higher order Katugampola fractional derivatives in the space $A C_{\rho}^{n}[a, b]$ defined below.

Moreover, in Section 4 we describe some properties of the Katugampola fractional operators. 


\section{Existence of the Katugampola fractional derivative}

Let $A C[a, b]$ be the space of functions $f$ which are absolutely continuous on $[a, b]$ i.e.,

$$
A C[a, b]:=\left\{f:[a, b] \rightarrow \mathbb{R}: f(t)=c+\int_{a}^{t} \varphi(s) d s\right\}
$$

where $c$ is an arbitrary real constant and $\varphi \in L[a, b]$. Definition (17) can be written as

$$
A C[a, b]:=\left\{f:[a, b] \rightarrow \mathbb{R}: f(t)=\tilde{c}-\int_{t}^{b} \varphi(s) d s\right\} .
$$

The existence of the Katugampola fractional derivative for $\alpha \in(0,1]$ was considered in [13, and case for $\alpha \in(1,2]$ in [4]. In this work, we consider the Katugampola fractional derivative for any positive real $\alpha$. For this purpose, let us introduce the space $A C_{\rho}^{n}[a, b]$, for $n \in \mathbb{N}$ which consists of those real-valued Lebesgue measurable functions $f$ on $(a, b)$ which have $\delta_{\rho}$-derivatives up to order $n-1$ and $\delta_{\rho}^{n-1} f$ is absolutely continuous on $[a, b]$ :

$$
A C_{\rho}^{n}[a, b]:=\left\{f:[a, b] \rightarrow \mathbb{R}: \delta_{\rho}^{n-1} f \in A C[a, b], \delta_{\rho}=t^{1-\rho} \frac{d}{d t}\right\} .
$$

If $\rho=1$ and $n=1$, the space $A C_{1}^{1}[a, b]$ coincides with $A C[a, b]$.

The space $A C_{\rho}^{n}[a, b]$ is characterized by the following result.

Lemma 3.1. Let $0<a<b<\infty, \rho>0$ and $n \in \mathbb{N}$. The space $A C_{\rho}^{n}[a, b]$ consists of those and only those functions $f$ which can be represented in the form

$$
f(t)=\sum_{i=0}^{n-1} c_{i}\left(\frac{t^{\rho}-a^{\rho}}{\rho}\right)^{i}+\frac{1}{(n-1) !} \int_{a}^{t}\left(\frac{t^{\rho}-\tau^{\rho}}{\rho}\right)^{n-1} \varphi(\tau) d \tau,
$$

or

$$
f(t)=\sum_{i=0}^{n-1} c_{i}(-1)^{i}\left(\frac{b^{\rho}-t^{\rho}}{\rho}\right)^{i}+\frac{(-1)^{n-1}}{(n-1) !} \int_{t}^{b}\left(\frac{\tau^{\rho}-t^{\rho}}{\rho}\right)^{n-1} \varphi(\tau) d \tau,
$$

where $c_{i}(i=0,1, \ldots, n-1)$ are arbitrary real constants and $\varphi \in L[a, b]$.

P r o o f. We will show only the proof of the formula (10). First, we prove necessity. Let $f \in A C_{\rho}^{n}[a, b]$. Then by (9) we have

$$
\delta_{\rho}^{n-1} f \in A C[a, b], \quad \text { where } \quad \delta_{\rho}=t^{1-\rho} \frac{d}{d t} .
$$


From (17) we get

$$
\delta_{\rho}^{n-1} f(t)=c_{n-1}+\int_{a}^{t} \varphi(\tau) d \tau
$$

where $c_{n-1}$ is an arbitrary constant and $\varphi \in L[a, b]$. In order to prove the formula (11), we should use the above (81) instead of (17) and the rest of the proof will be analogous. We can rewrite (12) in the form

$$
\frac{d}{d t} \delta_{\rho}^{n-2} f(t)=t^{\rho-1} c_{n-1}+t^{\rho-1} \int_{a}^{t} \varphi(\tau) d \tau .
$$

Now, integrating both sides of (13) we get

$$
\delta_{\rho}^{n-2} f(t)=c_{n-1} \frac{t^{\rho}-a^{\rho}}{\rho}+c_{n-2}+\int_{a}^{t} \frac{t^{\rho}-\tau^{\rho}}{\rho} \varphi(\tau) d \tau,
$$

where $c_{n-1}, c_{n-2}$ are arbitrary constants. Repeating this process $m(1 \leq m \leq$ $n-1)$ times we obtain successively

$$
\begin{aligned}
& \delta_{\rho}^{n-3} f(t)=c_{n-3}+c_{n-2} \frac{t^{\rho}-a^{\rho}}{\rho}+\frac{1}{2} c_{n-1}\left(\frac{t^{\rho}-a^{\rho}}{\rho}\right)^{2} \\
& +\frac{1}{2} \int_{a}^{t}\left(\frac{t^{\rho}-\tau^{\rho}}{\rho}\right)^{2} \varphi(\tau) d \tau \\
& \delta_{\rho}^{n-4} f(t)=c_{n-4}+c_{n-3} \frac{t^{\rho}-a^{\rho}}{\rho}+\frac{1}{2} c_{n-2}\left(\frac{t^{\rho}-a^{\rho}}{\rho}\right)^{2} \\
& +\frac{1}{6} c_{n-1}\left(\frac{t^{\rho}-a^{\rho}}{\rho}\right)^{3} \\
& +\frac{1}{6} \int_{a}^{t}\left(\frac{t^{\rho}-\tau^{\rho}}{\rho}\right)^{3} \varphi(\tau) d \tau \text {. } \\
& \delta_{\rho}^{n-m} f(t)=\sum_{i=0}^{m-1} c_{n-m+i}\left(\frac{t^{\rho}-a^{\rho}}{\rho}\right)^{i} \\
& +\frac{1}{(m-1) !} \int_{a}^{t}\left(\frac{t^{\rho}-\tau^{\rho}}{\rho}\right)^{m-1} \varphi(\tau) d \tau
\end{aligned}
$$

where $c_{n-1}, c_{n-2}, \ldots, c_{n-m}$ are arbitrary constants. Taking (14) with $m=n$, we obtain (10) and the necessity is proved. 


\section{PROPERTIES OF THE KATUGAMPOLA FRACTIONAL OPERATORS}

Now, let $f$ be represented by (10). Taking $\delta_{\rho}$-derivative $m$ times $(m \leq n-1)$, we have, respectively,

$$
\begin{aligned}
\delta_{\rho} f(t)= & \sum_{i=1}^{n-1} i c_{i}\left(\frac{t^{\rho}-a^{\rho}}{\rho}\right)^{i-1}+\frac{1}{(n-2) !} \int_{a}^{t}\left(\frac{t^{\rho}-\tau^{\rho}}{\rho}\right)^{n-2} \varphi(\tau) d \tau, \\
\delta_{\rho}^{2} f(t)= & \sum_{i=2}^{n-1} i(i-1) c_{i}\left(\frac{t^{\rho}-a^{\rho}}{\rho}\right)^{i-2}+\frac{1}{(n-3) !} \int_{a}^{t}\left(\frac{t^{\rho}-\tau^{\rho}}{\rho}\right)^{n-3} \varphi(\tau) d \tau, \\
\delta_{\rho}^{m} f(t)= & \sum_{i=m}^{n-1} c_{i} \frac{i !}{(i-m) !}\left(\frac{t^{\rho}-a^{\rho}}{\rho}\right)^{i-m} \\
& +\frac{1}{(n-m-1) !} \int_{a}^{t}\left(\frac{t^{\rho}-\tau^{\rho}}{\rho}\right)^{n-m-1} \varphi(\tau) d \tau .
\end{aligned}
$$

Thus, for $m=n-1$ we obtain

$$
\delta_{\rho}^{n-1} f(t)=c_{n-1}(n-1) !+\int_{a}^{t} \varphi(\tau) d \tau,
$$

which means that $f \in A C_{\rho}^{n}[a, b]$.

Theorem 3.2. Let $\rho>0, n \in \mathbb{N}, n-1<\alpha<n, 0<a<b<\infty$ and $f \in A C_{\rho}^{n}[a, b]$ then the Katugampola fractional derivatives $D_{a+}^{\alpha, \rho} f$ and $D_{b-}^{\alpha, \rho} f$ exist almost everywhere on $[a, b]$ and may be represented in the form

$$
\begin{aligned}
D_{a+}^{\alpha, \rho} f(t) & =\sum_{i=0}^{n-1} c_{i} \frac{\Gamma(i+1)}{\Gamma(i+1-\alpha)}\left(\frac{t^{\rho}-a^{\rho}}{\rho}\right)^{i-\alpha} \\
& +\frac{1}{\Gamma(n-\alpha)} \int_{a}^{t}\left(\frac{t^{\rho}-s^{\rho}}{\rho}\right)^{n-\alpha-1} \varphi(s) d s
\end{aligned}
$$

and

$$
\begin{aligned}
D_{b-}^{\alpha, \rho} f(t)= & \sum_{i=0}^{n-1} c_{i}(-1)^{i} \frac{\Gamma(i+1)}{\Gamma(i+1-\alpha)}\left(\frac{b^{\rho}-t^{\rho}}{\rho}\right)^{i-\alpha} \\
& +\frac{(-1)^{n-1}}{\Gamma(n-\alpha)} \int_{t}^{b}\left(\frac{s^{\rho}-t^{\rho}}{\rho}\right)^{n-\alpha-1} \varphi(s) d s
\end{aligned}
$$

where $c_{i}(i=0,1, \ldots, n-1)$ are some real constants and $\varphi \in L[a, b]$. 


\section{BARBARA ŁUPIŃSKA}

P r o o f. We show in detail only the proof of the formula (15). Since $f \in A C_{\rho}^{n}[a, b]$, we have the representation (10). Substituting it into the definition of the leftsided Katugampola fractional derivative and using (5), we obtain

$$
\begin{aligned}
D_{a+}^{\alpha, \rho} f(t)= & \sum_{i=0}^{n-1} c_{i} \frac{\Gamma(i+1)}{\Gamma(i+1-\alpha)}\left(\frac{t^{\rho}-a^{\rho}}{\rho}\right)^{i-\alpha} \\
& +\frac{\rho^{\alpha-2 n+1}}{(n-1) ! \Gamma(n-\alpha)} \delta_{\rho}^{n}\left(\int_{a}^{t} \frac{\rho \tau^{\rho-1}}{\left(t^{\rho}-\tau^{\rho}\right)^{1-n+\alpha}} \int_{a}^{\tau}\left(\tau^{\rho}-s^{\rho}\right)^{n-1} \varphi(s) d s d \tau\right) .
\end{aligned}
$$

Changing the order of integration we have

$$
\begin{aligned}
& \int_{a}^{t} \frac{\rho \tau^{\rho-1}}{\left(t^{\rho}-\tau^{\rho}\right)^{1-n+\alpha}} \int_{a}^{\tau}\left(\tau^{\rho}-s^{\rho}\right)^{n-1} \varphi(s) d s d \tau \\
& =\int_{a}^{t} \varphi(s) \int_{s}^{t} \frac{\rho \tau^{\rho-1}}{\left(t^{\rho}-\tau^{\rho}\right)^{1-n+\alpha}}\left(\tau^{\rho}-s^{\rho}\right)^{n-1} d \tau d s .
\end{aligned}
$$

The inner integral can be evaluated by the change of variable $u=\frac{\tau^{\rho}-s^{\rho}}{t^{\rho}-s^{\rho}}$. It gives

$$
\begin{aligned}
& \int_{s}^{t} \frac{\rho \tau^{\rho-1}}{\left(t^{\rho}-\tau^{\rho}\right)^{1-n+\alpha}}\left(\tau^{\rho}-s^{\rho}\right)^{n-1} d \tau \\
& =\left(t^{\rho}-s^{\rho}\right)^{2 n-\alpha-1} \int_{0}^{1} u^{n-1}(1-u)^{n-\alpha-1} d u .
\end{aligned}
$$

Using the Beta function and the fact (3), we obtain

$$
\begin{aligned}
& \int_{s}^{t} \frac{\rho \tau^{\rho-1}}{\left(t^{\rho}-\tau^{\rho}\right)^{1-n+\alpha}}\left(\tau^{\rho}-s^{\rho}\right)^{n-1} d \tau \\
& =\left(t^{\rho}-s^{\rho}\right)^{2 n-\alpha-1} \frac{\Gamma(n) \Gamma(n-\alpha)}{\Gamma(2 n-\alpha)} .
\end{aligned}
$$

Thus

$$
\begin{aligned}
& \int_{a}^{t} \frac{\rho \tau^{\rho-1}}{\left(t^{\rho}-\tau^{\rho}\right)^{1-n+\alpha}} \int_{a}^{\tau}\left(\tau^{\rho}-s^{\rho}\right)^{n-1} \varphi(s) d s d \tau \\
& =\frac{\Gamma(n) \Gamma(n-\alpha)}{\Gamma(2 n-\alpha)} \int_{a}^{t}\left(t^{\rho}-s^{\rho}\right)^{2 n-\alpha-1} \varphi(s) d s .
\end{aligned}
$$




\section{PROPERTIES OF THE KATUGAMPOLA FRACTIONAL OPERATORS}

Taking $\delta_{\rho}^{n}$-differentiation and using properties of Gamma function we obtain

$$
\begin{aligned}
& \delta_{\rho}^{n}\left(\frac{\Gamma(n) \Gamma(n-\alpha)}{\Gamma(2 n-\alpha)} \int_{a}^{t}\left(t^{\rho}-s^{\rho}\right)^{2 n-\alpha-1} \varphi(s) d s\right) \\
& =\frac{\rho \Gamma(n) \Gamma(n-\alpha)}{\Gamma(2 n-\alpha-1)} \delta_{\rho}^{n-1}\left(\int_{a}^{t}\left(t^{\rho}-s^{\rho}\right)^{2 n-\alpha-2} \varphi(s) d s\right) \\
& =\frac{\rho^{2} \Gamma(n) \Gamma(n-\alpha)}{\Gamma(2 n-\alpha-2)} \delta_{\rho}^{n-2}\left(\int_{a}^{t}\left(t^{\rho}-s^{\rho}\right)^{2 n-\alpha-3} \varphi(s) d s\right) \\
& =\cdots \\
& =\rho^{m} \Gamma(n) \Gamma(n-\alpha) \Gamma(2 n-\alpha-m) \delta_{\rho}^{n-m}\left(\int_{a}^{t}\left(t^{\rho}-s^{\rho}\right)^{2 n-\alpha-m-1} \varphi(s) d s\right) .
\end{aligned}
$$

Finally, taking $n=m$ we have

$$
\begin{aligned}
& \delta_{\rho}^{n}\left(\frac{\Gamma(n) \Gamma(n-\alpha)}{\Gamma(2 n-\alpha)} \int_{a}^{t}\left(t^{\rho}-s^{\rho}\right)^{2 n-\alpha-1} \varphi(s) d s\right) \\
& =\rho^{n} \Gamma(n)\left(\int_{a}^{t}\left(t^{\rho}-s^{\rho}\right)^{n-\alpha-1} \varphi(s) d s\right) .
\end{aligned}
$$

Substituting this into (17) and using the property of Gamma function (10), we obtain (15).

In order to prove the formula (16), we should start with substituting (11) into definition of the right-sided Katugampola fractional derivative. These details are left to the reader.

Corollary 3.3. If $0<\alpha<1$ and $f \in A C[a, b]$, then $D_{a+}^{\alpha, \rho} f$ exists almost everywhere in $[a, b]$ and

$$
D_{a+}^{\alpha, \rho} f(t)=c\left(\frac{\rho}{t^{\rho}-a^{\rho}}\right)^{\alpha}+\frac{1}{\Gamma(1-\alpha)} \int_{a}^{t}\left(\frac{\rho}{t^{\rho}-\tau^{\rho}}\right)^{\alpha} f^{\prime}(\tau) d \tau,
$$

where $c$ is real constant.

Remark 2. Relation (18) was proved in [13].

These results in Theorem 3.2 for the Katugampola fractional derivatives are analogous to those which have been proven for the Riemann-Liouville and the Hadamard fractional derivatives. It means that if we take in Theorem $3.2 \rho=1$, 


\section{BARBARA ŁUPIŃSKA}

we obtain the form of the Riemann-Liouville fractional derivatives of the order $\alpha$ in space $A C_{1}^{n}[a, b](n=[\alpha]+1)$, i.e.,

$$
\begin{gathered}
D_{a+}^{\alpha} f(t)=\sum_{i=0}^{n-1} c_{i}(t-a)^{i-\alpha}+\frac{1}{\Gamma(n-\alpha)} \int_{a}^{t} \frac{\varphi(s)}{(t-s)^{\alpha-n+1}} d s, \\
D_{b-}^{\alpha} f(t)=\sum_{i=0}^{n-1}(-1)^{i} c_{i}(b-t)^{i-\alpha}+\frac{(-1)^{n}}{\Gamma(n-\alpha)} \int_{t}^{b} \frac{\varphi(s)}{(s-t)^{\alpha-n+1}} d s,
\end{gathered}
$$

where $\varphi \in L[a, b]$ and $c_{i}$ are arbitrary constants. These relations were established in [11, Lemma 2.2]. Moreover, taking $\rho \rightarrow 0^{+}$in Theorem 3.2 we obtain result from [12, Theorem 3.4].

\section{Properties of the Katugampola fractional operators}

The Katugampola fractional integral satisfies several important properties including boundedness in the $L^{p}(a, b)$ space or linearity property. These facts were proved in [1. The following assertion shows that Katugampola fractional integrals satisfy semigroup property.

LEMMA 4.1. Let $\alpha>0, \beta>0, \rho>0$ and $f \in L[a, b]$ then

$$
I_{a+}^{\alpha, \rho} I_{a+}^{\beta, \rho} f(t)=I_{a+}^{\alpha+\beta, \rho} f(t),
$$

and

$$
I_{b-}^{\alpha, \rho} I_{b-}^{\beta, \rho} f(t)=I_{b-}^{\alpha+\beta, \rho} f(t)
$$

for all $t \in(a, b)$.

P r o o f. By definition of the Katugampola fractional integral, and next by changing order of integration we obtain

$$
I_{a+}^{\alpha, \rho} I_{a+}^{\beta, \rho} f(t)=\frac{\rho^{1-\alpha-\beta}}{\Gamma(\alpha) \Gamma(\beta)} \int_{a}^{t} s^{\rho-1} f(s) \int_{s}^{t} \frac{\rho \tau^{\rho-1}}{\left(t^{\rho}-\tau^{\rho}\right)^{1-\alpha}\left(\tau^{\rho}-s^{\rho}\right)^{1-\beta}} d \tau d s .
$$

Substitution $u=\frac{\tau^{\rho}-s^{\rho}}{t^{\rho}-s^{\rho}}$ in the inner integral gives

$$
I_{a+}^{\alpha, \rho} I_{a+}^{\beta, \rho} f(t)=\frac{\rho^{1-\alpha-\beta}}{\Gamma(\alpha) \Gamma(\beta)} \int_{a}^{t} s^{\rho-1} f(s)\left(t^{\rho}-s^{\rho}\right)^{\alpha+\beta-1} \int_{0}^{1} u^{\beta-1}(1-u)^{\alpha-1} d u d s .
$$

Using definition of the function Beta and its property (3), we obtain (19). The property (20) is proved by analogy. 


\section{PROPERTIES OF THE KATUGAMPOLA FRACTIONAL OPERATORS}

LEMMA 4.2. Let $\alpha>\beta>0, \rho>0$ and $f \in L[a, b]$ then

$$
D_{a+}^{\beta, \rho} I_{a+}^{\alpha, \rho} f(t)=I_{a+}^{\alpha-\beta, \rho} f(t), \quad a<t<b .
$$

P r o of. Let $n=[\beta]+1$. Using definitions of the Katugampola fractional operators and index rule (19) we obtain

$$
\begin{aligned}
D_{a+}^{\beta, \rho} I_{a+}^{\alpha, \rho} f(t) & =\delta_{\rho}^{n} I_{a+}^{n-\beta, \rho} I_{a+}^{\alpha, \rho} f(t) \\
& =\delta_{\rho}^{n} I_{a+}^{n, \rho} I_{a+}^{\alpha-\beta, \rho} f(t),
\end{aligned}
$$

which ends the proof, because $\delta_{\rho}^{n} I_{a+}^{n, \rho} f(t)=f(t)$, as it is mentioned in (44).

The next result shows that, for certain classes of functions, the Katugampola fractional derivative is the left inverse operator of the Katugampola fractional integral.

TheOREM 4.3. Let $\alpha>0, \rho>0$ and $f \in L[a, b]$, then

$$
D_{a+}^{\alpha, \rho} I_{a+\rho}^{\alpha, \rho} f(t)=f(t) .
$$

However, as we show below, the Katugampola fractional derivative is not the right inverse of the Katugampola fractional integral.

Theorem 4.4. Let $n-1<\alpha<n, n \in \mathbb{N}, \rho>0, I_{a+}^{n-\alpha, \rho} f \in A C_{\rho}^{n}[a, b]$, then

$$
I_{a+}^{\alpha, \rho} D_{a+}^{\alpha, \rho} f(t)=f(t)+\sum_{i=0}^{n-1} \tilde{c}_{i}\left(\frac{t^{\rho}-a^{\rho}}{\rho}\right)^{i-n+\alpha},
$$

where $\tilde{c}_{i}$ are real constants.

P r o of. From definition of the space $A C_{\rho}^{n}[a, b]$ (9) and Lemma 3.1, we can rewrite the assumption $I_{a+}^{n-\alpha, \rho} f \in A C_{\rho}^{n}[a, b]$ as

$$
\delta_{\rho}^{n-1} I_{a+}^{n-\alpha, \rho} f(t)=c+\int_{a}^{t} \varphi(\tau) d \tau
$$

and

$$
I_{a+}^{n-\alpha, \rho} f(t)=\sum_{i=0}^{n-1} c_{i}\left(\frac{t^{\rho}-a^{\rho}}{\rho}\right)^{i}+\frac{1}{(n-1) !} \int_{a}^{t}\left(\frac{t^{\rho}-\tau^{\rho}}{\rho}\right)^{n-1} \varphi(\tau) d \tau,
$$

where $c_{i}$ are real constants and $\varphi \in L[a, b]$.

Taking $\delta_{\rho}$-derivative of (21) we obtain

$$
D_{a+\rho}^{\alpha, \rho} f(t)=t^{1-\rho} \varphi(t) .
$$

Thus

$$
I_{a+}^{\alpha, \rho} D_{a+}^{\alpha, \rho} f(t)=I_{a+}^{\alpha, \rho}\left[t^{1-\rho} \varphi(t)\right] .
$$


On the other hand, applying operator $D_{a+}^{n-\alpha, \rho}$ to both sides of (22) we have

$$
\begin{aligned}
f(t)= & \sum_{i=0}^{n-1} c_{i} D_{a+}^{n-\alpha, \rho}\left(\frac{t^{\rho}-a^{\rho}}{\rho}\right)^{i} \\
& +\frac{1}{(n-1) !} D_{a+}^{n-\alpha, \rho}\left(\int_{a}^{t}\left(\frac{t^{\rho}-\tau^{\rho}}{\rho}\right)^{n-1} \varphi(\tau) d \tau\right) .
\end{aligned}
$$

From (5) we have

$$
D_{a+}^{n-\alpha, \rho}\left(\frac{t^{\rho}-a^{\rho}}{\rho}\right)^{i}=\frac{\Gamma(i+1)}{\Gamma(i+1-n+\alpha)}\left(\frac{t^{\rho}-a^{\rho}}{\rho}\right)^{i-n+\alpha} .
$$

Furthermore, we have

$$
\begin{aligned}
& D_{a+}^{n-\alpha, \rho}\left(\int_{a}^{t}\left(\frac{t^{\rho}-\tau^{\rho}}{\rho}\right)^{n-1} \varphi(\tau) d \tau\right) \\
& =\delta_{\rho} I_{a+}^{1-n+\alpha, \rho}\left[\int_{a}^{t}\left(\frac{t^{\rho}-\tau^{\rho}}{\rho}\right)^{n-1} \varphi(\tau) d \tau\right] \\
& =\frac{\rho^{-\alpha}}{\Gamma(1-n+\alpha)} \delta_{\rho}\left(\int_{a}^{t} \varphi(s) \int_{s}^{t} \frac{\rho \tau^{\rho-1}\left(\tau^{\rho}-s^{\rho}\right)^{n-1}}{\left(t^{\rho}-\tau^{\rho}\right)^{n-\alpha}} d \tau\right) .
\end{aligned}
$$

Substitution $u=\frac{\tau^{\rho}-s^{\rho}}{t^{\rho}-s^{\rho}}$ gives

$$
\begin{aligned}
& D_{a+}^{n-\alpha, \rho}\left(\int_{a}^{t}\left(\frac{t^{\rho}-\tau^{\rho}}{\rho}\right)^{n-1} \varphi(\tau) d \tau\right) \\
& =\frac{\rho^{-\alpha}}{\Gamma(1-n+\alpha)} \delta_{\rho}\left(\int_{a}^{t} \varphi(s)\left(t^{\rho}-s^{\rho}\right)^{\alpha} \int_{0}^{1} u^{n-1}(1-u)^{\alpha-n} d u d s\right) .
\end{aligned}
$$

Finally, using properties of Beta and Gamma functions we obtain

$$
\begin{aligned}
D_{a+}^{n-\alpha, \rho}\left(\int_{a}^{t}\left(\frac{t^{\rho}-\tau^{\rho}}{\rho}\right)^{n-1} \varphi(\tau) d \tau\right) & =\frac{\rho^{-\alpha} \Gamma(n)}{\Gamma(\alpha+1)} \delta_{\rho}\left(\int_{a}^{t} \varphi(s)\left(t^{\rho}-s^{\rho}\right)^{\alpha} d s\right) \\
& =\Gamma(n) I_{a+}^{\alpha, \rho}\left(t^{1-\rho} \varphi(t)\right) .
\end{aligned}
$$




\section{PROPERTIES OF THE KATUGAMPOLA FRACTIONAL OPERATORS}

Substituting (25) and (26) into (24) we have

$$
f(t)=\sum_{i=0}^{n-1} c_{i} \frac{\Gamma(i+1)}{\Gamma(i+1-n+\alpha)}\left(\frac{t^{\rho}-a^{\rho}}{\rho}\right)^{i-n+\alpha}+I_{a+}^{\alpha, \rho}\left(t^{1-\rho} \varphi(t)\right) .
$$

By (23) and (27) we get the thesis, and the proof is completed.

The above theorem allows to find a general solution of the following fractional equation

$$
D_{a+}^{\alpha, \rho} u(t)=0
$$

where $\alpha, \rho$ are positive real constants. We have

Theorem 4.5. Let $n-1<\alpha<n, n \in \mathbb{N}$ and $\rho>0$, then equation (28) holds if, and only if,

$$
u(t)=\sum_{i=1}^{n} c_{i}\left(\frac{t^{\rho}-a^{\rho}}{\rho}\right)^{\alpha-i}
$$

The proof of this theorem is trivial, so it is left to the reader.

\section{REFERENCES}

[1] KATUGAmPOLA, U. N.: New approach to a generalized fractional integral, Appl. Math. Comput. 218 (2011), 860-865.

[2] KATUGAMPOLA,U. N.: A new approach to generalized fractional derivatives, Bull. Math. Anal. App. 6 (2014), 1-15.

[3] AKKURT, A.-KAÇAR, Z.-YILDIRIM, H.: Generalized fractional integral inequalities for continuous random variables, J. Probab. Stat. (2015), Paper no. 958980, 7 pp.

[4] ŁUPIŃSKA, B.-ODZIJEWICZ, T.: A Lyapunov-type inequality with the Katugampola fractional derivative, Math. Methods Appl. Sci. 41 (2018), no. 18, 8985-8996.

[5] CHEN, H.-KATUGAMPOLA, U. N.: Hermite-Hadamard and Hermite-Hadamard-Fejér type Inequalities for Generalized Fractional Integrals, J. Math. Anal. Appl. 446 (2017), no. 2, 1274-1291.

[6] ŁUPIŃSKA, B.-ODZIJEWICZ, T.-SCHMEIDEL, E.: On the solutions to a generalized fractional Cauchy problem, Appl. Anal. Discr. Math. 10 (2016), no. 2, 332-344.

[7] ZENG, S.- BALEANU, D.-BAI, Y.-WU, G.: Fractional differential equations of Caputo-Katugampola type and numerical solutions, Appl. Math. Comput. 315 (2017), 549-554.

[8] KATUGAMPOLA,U. N.: Mellin transforms of the generalized fractional integrals and derivatives, Appl. Math. Comput. 257 (2015), 566-580.

[9] CAO, L.-KONG, H.-ZENG, S. D.: Maximum principles for time-fractional Caputo-Katugampola diffusion equations, J. Nonlinear Sci. Appl. 10 (2017), 2257-2267. 


\section{BARBARA ŁUPIŃSKA}

[10] BALEANU, D.-WU, G. C.-ZENG, S. D.: Chaos analysis and asymptotic stability of generalized Caputo fractional differential equations, Chaos Solitons Fractals 102 (2017), 99-105.

[11] KILBAS, A. A.-SRIVAStaVA, H. M.-TRUJILlO, J. J.: Theory and Applications of Fractional Differential Equations, Elsevier, Amsterdam, 2006.

[12] KILBAS, A. A.: Hadamard-type fractional calculus, J. Korean Math. Soc. 38(6) (2001), 1191-1204.

[13] COTTOne, G.-MALinOWSKA, A. B.-ODZIJEWICZ, T.: The non-homogeneous Voigt-Katugampola model of visco-elastic material (to appear)

[14] ŁUPIŃSKA, B.-ODZIJEWICZ, T.-SCHMEIDEL, E.: Some properties of generalized fractional integrals and derivatives, In: Proceedings of the International Conference of Numerical Analysis and Applied Mathematics 2016 (ICNAAM-2016) Book Series: AIP Conference Proceedings.

Received October 21, 2020

Insitute of Computer Science

of University of Biatystok

Konstantego Ciotkowskiego $1 \mathrm{M}$

$P L-15-245$ Biatystok

POLAND

E-mail: bpietruczuk @math.uwb.edu.pl 